\title{
Studies on the biodegradation of natural and synthetic polyethylene by Pseudomonas spp
}

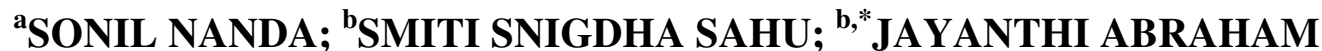

\author{
${ }^{a}$ Orissa University of Agriculture \& Technology, Bhubaneswar 751 003, Orissa, India \\ ${ }^{\mathrm{b}}{ }^{*}$ School of Bio Sciences and Technology, VIT University, Vellore 632 014, Tamil Nadu, India
}

\begin{abstract}
The current article makes a comparative analysis between the biodegradation of natural and synthetic polyethylene by three different species of Pseudomonas. The three Pseudomonas spp. (P1, P2, and P3) were indigenous to locations: (1) domestic waste disposal site dumped with household garbage and vegetable waste; (2) soil from textile effluents drainage site; and (3) soil dumped with sewage sludge, respectively. The ability of these species in degrading natural and synthetic polyethylene was investigated. Pure culture shake-flask incubation for 8 weeks was performed for the purpose of biodegradation. The natural or biodegradable polyethylene used in the study was disposable plastic bags containing $6 \%$ vegetable starch. The initial and final dry weights of plastic bags before and after incubation in the culture medium were compared and the percentage of degradation was calculated. Among all the treatments, Pseudomonas sp. from sewage sludge dump (P1) was found to degrade polyethylene efficiently with $46.2 \%$ for natural and $29.1 \%$ for synthetic polyethylene. In contrast, Pseudomonas sp. from household garbage dump (P2) gave the lowest biodegradability of $31.4 \%$ and $16.3 \%$ for natural and synthetic polyethylene, respectively. However, Pseudomonas sp. isolated from textile effluents drainage site gave an intermediate biodegradability of $39.7 \%$ and $19.6 \%$ for natural and synthetic polyethylene, respectively. Overall, natural polyethylene gave a rapid biodegradation within the same duration than the synthetic ones. The active enzymes produced by the bacteria caused mechanical denting and weight loss in polyethylene. @ JASEM
\end{abstract}

Polyethylene is a synthetic polymer made of long chain monomers of ethylene. It is a thermoplastic commodity mostly used for packaging. About 140 million tonnes of synthetic polymers are produced worldwide annually with their utility escalating at a rate of $12 \%$ per annum (Shimao, 2001). With such massive amount of polyethylene manufactured it is a tedious job to seek for their efficient disposal. The durability, light weight, and processability of these polymers causes them to linger in the nature for centuries and end up in landfills and natural water resources creating a severe threat to the environment and its ecosystems (Hoffmann et al., 2003; Jang et al., 2002). In order to manage the utility of these polymers in the nature, there are two ways: one is to exploit the microorganisms in degrading polyethylene and the other is to develop artificial polymers susceptible to biodegradation. Subsequently, to gain large-scale acceptance these man-made biodegradable polyethylene should retain all the essential properties of utility by the consumer and when discarded in the environment should demonstrate their degradability more rapidly than the conventional ones (El-Shafei et al., 1998). Unlike most polymers, biodegradable polymers when disposed favourably in the environment (e.g. compost, soil, or waste water) are acted upon and utilized by the indigenous microorganisms as sources of carbon and energy, thus are degraded (Starnecker and Menner, 1996). As new biodegradable polymers and their packaging applications are emerging, there is a need to address their environmental performance particularly the time required for their complete disintegration in nature (Kale et al., 2007). Less often it happens that the polymer may be safe before biodegradation but may turn toxic during degradation (Delgi-Innocenti et al., 2001). Today there is a growing interest in the development of biodegradable plastics that would enhance the degradability of other plastic products in landfills and composts under natural conditions (Pometto et al., 1992).

Microbial degradation of plastics is caused by enzymatic activities leading to a chain cleavage of the polymer into oligomers and monomers after which they are further metabolized by the microbial cells. Aerobic metabolism results in carbon dioxide and water (Starnecker and Menner, 1996), whereas anaerobic metabolism results in carbon dioxide, water, and methane as the end products, respectively (Gu et al., 2000). The depolymerisation results due to various physical or biological forces (Swift, 1997). The physical forces such as temperature, moisture, pressure etc. cause mechanical damage to the polymer so that the biological forces like enzymes and other microbial metabolites catalyze the process. Synthetic plastics, also known as petroplastics, persist in the nature after their disposal for a longer period with very less deterioration. They are often found in soils, agricultural fields, water resources etc. and are a cause for death of many animals and fishes for being swallowed as polyethylene debris (Yang et al., 2004). Alternatively, with modern eco-friendly technologies, biodegradable plastics or bioplastics have been developed from natural products. Unlike synthetic polyethylene that are derived from petroleum products, biopolyethylene are produced from renewable biomass sources such as vegetable extracts, sugar cane bagasse, food starch, agricultural by-products, organic minerals etc.

This research article evaluates the biodegradability of natural and synthetic polyethylene by three different Pseudomonas spp. isolated from variant locations in laboratory conditions by a pure culture shake-flask incubation method. The percent of biodegradation was evaluated by comparing the initial and final dry weights of polyethylene before and after incubation in their respective culture media. Our hypothesis and purpose of selecting Pseudomonas spp. for 
biodegradation was, this bacteria are predominant in nature and are often found to survive in nutrient deficient ecosystems with their versatile metabolism, hence they would be able to utilize polyethylene amended in the nutrient medium as carbon and energy source when basal nutrients in the medium are exhausted.

\section{MATERIALS AND METHODS}

Isolation of polyethylene degrading bacteria: Soil sample from three different locations were collected to isolate Pseudomonas and compare their biodegradability. Locations chosen were (1) domestic waste disposal site dumped with household garbage and vegetable waste, (2) soil from textile effluents drainage site, and (3) soil dumped with sewage sludge. The soil sample was analysed for its indigenous bacterial enumeration and isolation purpose. Selective isolation of Pseudomonas spp. were performed and the isolates (P1, P2, and P3) were obtained in pure culture to be used as inoculum in degrading polyethylene. The cultures were maintained at ambient temperature and frequently revived to sustain its viability status. Isolation of Pseudomonas spp. from the soil was done by standard dilution methods and inoculation on King's medium B agar. After incubation, the colonies were studied morphologically and biochemically. The characteristic type of growth on solid media under appropriate cultural condition and colony morphology was used in presumptive identification. Colony characters and Gram staining, and motility were recorded for morphological characters, whereas biochemical parameters such as indole, methyl red, Voges Proskauer, citrate utilization, urease, nitrate reduction, sugar fermentation, triple sugar iron, hydrogen sulphide production, oxidase, catalase, and denitrification tests were performed.

Selection of polyethylene bags: Polyethylene bags (both natural and synthetic) each having 50 microns thickness were used for comparative investigation of their biodegradability nature. The natural polyethylene bag used was made from a combination of virgin plastic vegetable starch $(6 \%)$, organic minerals, and vegetable extracts, whereas the synthetic polyethylene bag was of conventional plastic polymer of petroleum origin.

Pre-treatment of polyethylene:The polyethylene bags were cut in small strips and transferred to a fresh solution containing $70 \mathrm{ml}$ Tween $80,10 \mathrm{ml}$ bleach, and $983 \mathrm{ml}$ distilled water and stirring for 30 to 60 minutes (El-Shafei et al., 1998). The strips were transferred to a beaker with distilled water and stirred for 1 hour. Further, they were aseptically relocated to ethanol solution $70 \% \mathrm{v} / \mathrm{v}$ for $30 \mathrm{~min}$. Finally, the polyethylene strips were transferred to a Petri dish and incubated at 45 to $50^{\circ} \mathrm{C}$ overnight. Ethanol was used to disinfect the polyethylene and remove any organic matter adhering to its surface.

Treatment of polyethylene: The isolated Pseudomonas spp. (P1, P2, and P3) were inoculated in $200 \mathrm{ml}$ of nutrient broth in conical flasks with $0.5 \mathrm{~g}$ of the polyethylene strips (natural and synthetic) amended to each flask except the blank. All the treatments were incubated in an incubator shaker at $150 \mathrm{rpm}$ for 8 weeks at $40^{\circ} \mathrm{C}$. The experimental design for the three Pseudomonas spp. comprised of two treatments each i.e. T1 (Pseudomonas + natural polyethylene) and T2 (Pseudomonas + synthetic polyethylene) with controls i.e. C1 (natural polyethylene) and C2 (synthetic polyethylene) and blank (Pseudomonas). A blank for every Pseudomonas spp. was used to examine the viability of the bacteria and check for any contamination.

Dry weight determination of recovered polyethylene: The residual polyethylene particles were recovered from the broth cultures. The bacterial biofilm and cell mass adhering to the polyethylene surface was washed by a $2 \%(\mathrm{v} / \mathrm{v})$ aqueous sodium dodecyl sulphate solution for 2 to 3 hours and finally with distilled water (Hadad et al., 2005). The washed polyethylene particles were air-dried and weighed. The dry weights of recovered polyethylene from the culture media were taken in weekly intervals (i.e. day 0 , day 7 , day 14 , day 21 , day 28 , day 35 , day 42 , day 49, and day 56) for accounting the rate of biodegradation.

\section{RESULTS AND DISCUSSION}

The Pseudomonas spp. from three diverse terrestrial locations (P1: household garbage dump; P2: soil from textile effluents drainage site; and P3: sewage sludge dump) were isolated and characterised basing on their morphological and biochemical characteristics (Table 1). After 8 weeks of incubation in an incubator shaker at $40^{\circ} \mathrm{C}$ and $150 \mathrm{rpm}$, the culture flasks were removed and tested for the viability of bacteria and any contamination in the medium. The blanks and the treatments showed good stability of Pseudomonas in the medium with no sign of contamination. In addition, there was intense biofilm formation by all the Pseudomonas spp. which was clearly visible in the broth. Among all the treatments, P3 (i.e. Pseudomonas from sewage sludge dump) was found to degrade both natural and synthetic polyethylene very efficiently with $46.2 \%$ for T1 (Pseudomonas + natural polyethylene) and $29.1 \%$ for T2 (Pseudomonas + synthetic polyethylene). In contrast, P1 (i.e. Pseudomonas from household garbage dump) gave the lowest biodegradability of $31.4 \%$ for $\mathrm{T} 1$ and $16.3 \%$ for T2. However, P2 (i.e. Pseudomonas isolated from textile effluents drainage soil) gave an intermediate biodegradability of $39.7 \%$ and $19.6 \%$ for $\mathrm{T} 1$ and $\mathrm{T} 2$, respectively. 
Table 1. Morphological and biochemical characteristics of Pseudomonas spp. isolated from household garbage dump, textile industrial effluents dump, and sewage sludge dump

\begin{tabular}{|l|c|c|c|}
\hline \multirow{2}{*}{ Characteristics } & \multicolumn{3}{|c|}{ Pseudomonas } \\
\cline { 2 - 4 } & P1 & P2 & P3 \\
\hline Colony color & $\begin{array}{c}\text { Bluish } \\
\text { green }\end{array}$ & Green & $\begin{array}{c}\text { Greenish } \\
\text { yellow }\end{array}$ \\
\hline Gram staining & - & - & - \\
\hline Motility & + & + & + \\
\hline Indole & - & - & - \\
\hline Methyl red & - & - & - \\
\hline Voges Proskauer & - & - & - \\
\hline Citrate & + & + & + \\
\hline Urease & + & + & + \\
\hline Nitrate & + & - & - \\
\hline Triple sugar iron & $\mathrm{k} / \mathrm{k}$ & $\mathrm{k} / \mathrm{k}$ & $\mathrm{k} / \mathrm{k}$ \\
\hline $\begin{array}{l}\text { Hydrogen } \\
\text { sulphide }\end{array}$ & - & - & - \\
\hline Catalase & + & + & + \\
\hline Oxidase & + & + & + \\
\hline Denitrification & + & + & - \\
\hline
\end{tabular}

The trend in the biodegradation of the plastic bags (natural and synthetic) represented by P1, P2, and P3 with respect to the incubation time in weekly intervals from day 0 to day 56 are presented in Figures 1, 2, and 3 . Within all treatments, T1 presented more or less consistent biodegradation, whereas T2 was little meander. The natural polyethylene contained vegetable starch of $6 \%$ which could have enhanced the biodegradation rate from the initial phase of experimental trial. In addition to the basal nutrients of the broth, Pseudomonas spp. might have utilized the vegetable extracts and other organic components from the natural polyethylene which may be another reason of this uniformity of biodegradation. Nevertheless, the viability of Pseudomonas in the broth on day 56 suggested that the organism was still in the log phase. This explains that the natural polyethylene provided sufficient carbon and energy source to the bacteria for growth and multiplication. P3 showed maximum biodegradability of both the polyethylene bags and virtually $50 \%$ degradation of natural ones. The reason might be the metabolism of the particular isolate. As this Pseudomonas sp. is isolated from a location with rich organic nutrients from sewage sludge, it may have the ability to utilize utmost organic carbon. Therefore, natural polyethylene served the bacteria with its additional vegetable starch ingredient which led them to consume natural polyethylene more rapidly than the synthetic ones. With our findings, we assume that the bacteria were able to utilize natural polymers faster than the synthetic ones because less metabolic activities are sufficient enough to dissociate the carbon backgrounds of natural polyethylene than the complex enzymatic reactions that are required for synthetic polymers.

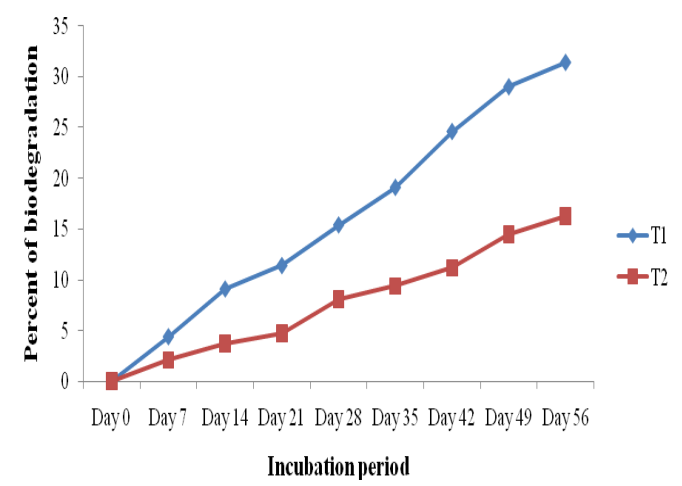

Figure 1. Trend of biodegradation of polyethylene (natural and synthetic) by P1 (Pseudomonas from household garbage dump)

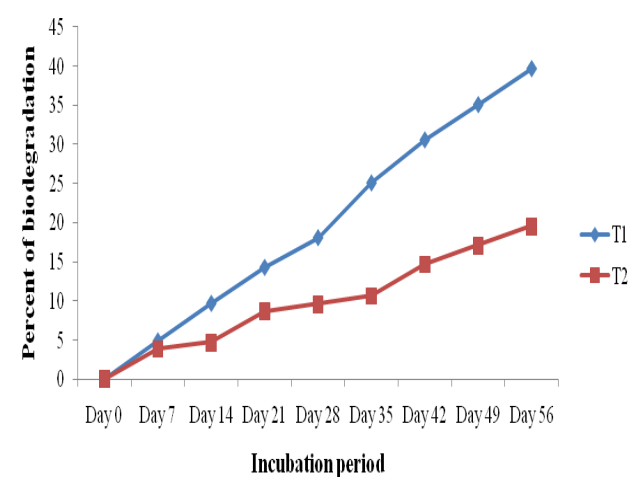

Figure 2. Trend of biodegradation of polyethylene (natural and synthetic) by P2 (Pseudomonas from textile effluents drainage soil)



Figure 3. Trend of biodegradation of polyethylene (natural and synthetic) by P3 (Pseudomonas from sewage sludge dump)

In contrast, the viability status of $\mathrm{P} 1$ in $\mathrm{T} 2$ on day 56 gave a little or no sign of multiplication which implies that the bacteria would have been in the stationary phase. This correlates to the availability of nutrients in the medium. The degradation rate was almost steady until day 21 after which it took a sudden leap. It might be assumed that Pseudomonas sp. metabolized the available basal media nutrients 
for about 3 weeks and after their limitation, it geared up to utilize the carbon sources from the polyethylene.

During the incubation period, the system was maintained undisturbed with no supplementation of exogenous nutrients into the medium. Hence, it may be established that after the mineral nutrients from the media were completely utilized by the bacteria, polyethylene served as the carbon and energy source. Moreover, the active enzymes produced by Pseudomonas catalyzed the degradation process and the biofilm aided them to adhere to the polyethylene surface rather than being solitary in the liquid media. Whatever the rationale may be, biodegradation depends upon polymer characteristics, organism type and nature of pre-treatment (Shah et al., 2008).

With such a wide array of application, the world has always seemed to be wrapped in plastics. Moreover, with their environmental impact evoking global concern and the petrochemical resources being limited it is now leading the humankind to search for an alternative resource i.e. the plant-based polymer. The significant research in biodegradation of polyethylene in the last few decades have increased our knowledge of degrading microorganisms, their enzymes, and the type of polymer best degrading under natural conditions. This enhances our understanding in developing new technologies or modifying the existing ones to degrade the plastics in an environmental-friendly way to no toxic byproducts. Recently, biodegradable plastics have gained much scientific interest but their commercial awareness is exceedingly required. The issue of synthetic plastic utility and their waste ending up in the environment can be partly resolved by developing and subsequently applying biodegradable materials (Dřímal et al., 2007). Hence, the future attention is on the commercial development and application of natural and eco-friendly polyethylene.

\section{REFERENCES}

Delgi-Innocenti, F; Bellia, G; Tosin, M; Kapanen, A; Itävaara, M (2001). Detection of toxicity released by biodegradable plastics after composting in activated vermiculite. Polym. Degrad. Stab. 73: $101-106$.

Dřímal, P; Hoffmann, J; Družbík, M (2007). Evaluating the aerobic biodegradability of plastics in soil environment through GC and IR analysis of gaseous phase. Polym. Test. 26: 729 - 751.

El-Shafei, HA; El-Nasser, NHA; Kansoh, AL; Ali, AM (1998). Biodegradation of disposable polyethylene by fungi and Streptomyces species. Polym. Degrad. Stab. 62: 361 - 365.
Gu, JD; Ford, TE; Mitton, DB; Mitchell, R (2000). Microbial corrosion of metals. The Uhlig Corrosion Handbook. 2nd Edition. Wiley, New York, USA.

Hadad, D; Geresh, S; Sivan, A (2005). Biodegradation of polyethylene by the thermophilic bacterium Brevibacillus borstelensis. J. Appl. Microbiol. 98: 1093 - 1100.

Hoffmann, J; Řezníčková, I; Kozáková, J; Růžička, J; Alexy, P; Bakoš, D; Precnerová, L (2003). Assessing biodegradability of plastic based on poly(vinyl alcohol) and protein wastes. Polym. Degrad. Stab. 79: 511 - 519.

Jang, J-C; Shin, P-K; Yoon, J-S; Lee, I-M; Lee, H-S; Kim, M-N (2002). Glucose effects on the biodegradation of plastics by compost from food garbage. Polym. Degrad. Stab. 76: 155 - 159.

Kale, G; Auras, R; Singh, SP; Narayan, R (2007). Biodegradability of polylactide bottles in real and stimulated composting conditions. Polym. Test. 26: 1049 - 1061 .

Pometto, AL; Lee, B; Johnson, KE (1992). Production of an Extracellular PolyethyleneDegrading Enzyme(s) by Streptomyces Species. Appl. Environ. Microbiol. 58 (2): 731 - 733.

Shah, AA; Hasan, F; Hameed, A; Ahmed, S (2008). Biological degradation of plastics: A Comprehensive review. Biotechnol. Adv. 26: 246 -265 .

Shimao, M (2001). Biodegradation of plastics. Curr. Opin. Biotechnol. 12: 242 - 247.

Starnecker, A; Menner, M (1996). Assessment of biodegradability of plastics under stimulated composting conditions in a laboratory test system. Int. Biodeterior. Biodegrad. 85 - 92.

Swift, G (1997). Non-medical biodegradable polymers: environmentally degradable polymers. Handbook of biodegradable polymers. Harwood Acedemic, Amsterdam.

Yang, H-S; Yoon, J-S; Kim, M-N (2004). Effects of storage of a mature compost on its potential for biodegradation of plastics. Polym. Degrad. Stab. 84: 411 - 417. 